\title{
Adrenal Gland Neoplasm
}

National Cancer Institute

\section{Source}

National Cancer Institute. Adrenal Gland Neoplasm. NCI Thesaurus. Code C2859.

A benign or malignant (primary or metastatic) neoplasm affecting the adrenal glands. 